\title{
Hydration water structure, hydration forces, and mechanical properties of polysaccharide films
}

Michael Grossutti and John R. Dutcher*

Department of Physics, University of Guelph, Guelph, ON, Canada N1G 2W1

\section{Supporting Information}

This document contains a supporting data figure.

* correspondence to: dutcher@uoguelph.ca 


\section{Equilibration of Films for Ellipsometry and ATR-FTIR Experiments}

We have measured the time dependence of the ellipsometry experiment in response to abrupt changes in RH (Figure S1). These data indicate that saturation of the ellipsometry signal is achieved after $\sim 250 \mathrm{~s}$. In fact, equilibration of the polysaccharide film likely occurs more quickly and the time dependence in the measured curves just corresponds to the time for the sample chamber to reach equilibrium following the RH change. The data also shows the reversibility of the swelling process for abrupt increases and decreases in RH. In addition, we recently combined ellipsometry data (ultrathin film samples equilibrated for $20 \mathrm{~min}$ using the same protocol as in the present study) and gravimetric analysis data (small mass samples equilibrated for 14 days), for PG in the hydration force regime. ${ }^{48} \mathrm{We}$ measured excellent overlap of the ellipsometry and gravimetric analysis data, indicating that the ellipsometry samples were sufficiently equilibrated.

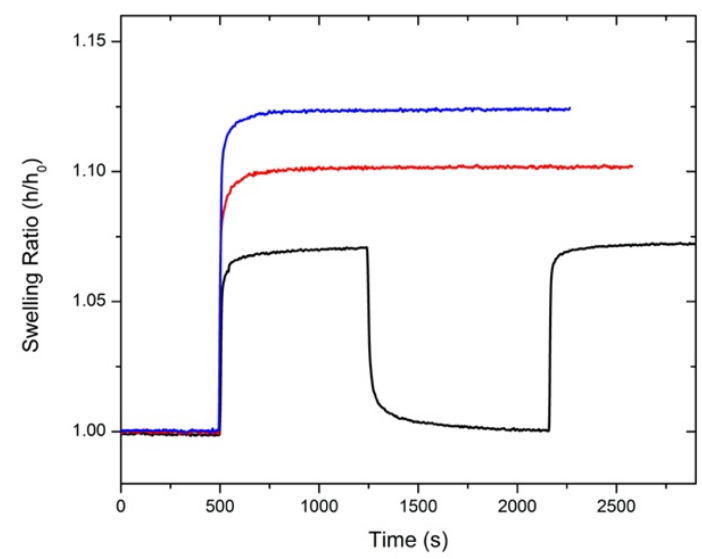

Figure S1: Time dependence of the swelling ratio $\left(h / h_{0}\right)$, as measured using ellipsometry for a PG film of dry thickness $55 \mathrm{~nm}$, for jumps in RH: $0 \%$ to $58 \%$ (blue curve), $0 \%$ to $50 \%$ (red curve), and cycling between $\mathrm{RH}=0 \%$ and $35 \%$ (black curve). 
For the ATR-FTIR experiments, it is necessary for the water to penetrate through the 3micrometer thick film down to the Ge crystal surface. To address this, we have performed deuterium exchange measurements with $\mathrm{D}_{2} \mathrm{O}$ vapour that showed that the entire IR film is accessible to solvent, with nearly all protons exchanged within 5 min (SI of ref. 6). In the present study, for both ellipsometry and ATR-FTIR experiments, we measured the response of the films to changes in $\mathrm{RH}$ for more than hour and found that 20 min was sufficient time to reach equilibrium for both the thicker IR films and ultrathin ellipsometry films. 